\title{
Use of Balanced Steady-State Free Precession Sequences in Evaluation of Drop Metastases
}

$\mathbf{R}_{\mathrm{tratan}}^{\mathrm{e}}$ ecently, an overseas colleague had reached out to us, having trouble implementing the sequences suggested by Buch et al ${ }^{1}$ for their patients in pediatric oncology. We believe that the issue was rooted in some inaccuracies relating to the sequences described in the article. We are writing this letter to clarify the terminology and sequence families for those who wish to use these classes of sequences. This letter by no means reduces the value of the article. We are quite happy to see that Buch et al have done a formal evaluation of the potential advantages of these sequences in further evaluation of drop metastases over previous anecdotal evidence.

$3 \mathrm{D}$ driven equilibrium pulses typically are not relevant to or applied in balanced steady-state free precession (bSSFP), which is a gradient recalled-echo sequence. These pulses use a $-90^{\circ}$ flip of the spins to enhance the T1 recovery of CSF, for example, in short-TR 3D fast spin-echo sequences. ${ }^{2}$ This results in a bright CSF signal in relatively short time, though it does not have true T2-weighting.

In addition, regular bSSFP sequences (also known as true fast imaging with steady-state precession [TruFISP], FIESTA, and balanced fast-field echo [bFFE] by various manufacturers) will not work well for the reported intended purposes of Buch et $\mathrm{al}^{1}$ due to phase shift errors and banding, particularly considering the long TR of 3D sequences. ${ }^{3}$ However, a further modification of bSSFP needs to be made using 2 back-to-back sequence pairs with and without phase alterations and using maximum intensity projection at the end to cancel the phase errors, hence the longer acquisition times. This additional modification has been implemented as FIESTA-C (not FIESTA) and CISS and is the one to which the authors would have been referring. ${ }^{4}$

http://dx.doi.org/10.3174/ajnr.A5964
On another note, one must consider that the presumed benefits of using these balanced SSFP sequences compared with T1weighted images is highly dependent on how the baseline T1weighted images are acquired and if other sequences, such as high-quality T2-weighted sequences, are used in the protocol as well. Having more than one plane postcontrast T1-weighted image, using newer thin-section T1-weighted images, and combining them with relatively higher resolution T2-weighted images will likely dampen the advantages of bSSFP because they will no longer be compared with a limited single-plane spin-echo T1weighted sequence. Nevertheless, using high-resolution bSSFPderived sequences can certainly be useful.

\section{REFERENCES}

1. Buch K, Caruso P, Ebb D, et al. Balanced steady-state free precession sequence (CISS/FIESTA/3D driven equilibrium radiofrequency reset pulse) increases the diagnostic yield for spinal drop metastases in children with brain tumors. AJNR Am J Neuroradiol 2018;39:1355-61 CrossRef Medline

2. Melhem ER, Itoh R, Folkers PJ. Cervical spine: three-dimensional fast spin-echo MR imaging-improved recovery of longitudinal magnetization with driven equilibrium pulse. Radiology. 2001;218:283-88 CrossRef Medline

3. Bieri O, Scheffler K. Fundamentals of balanced steady state free precession MRI. J Magn Reson Imaging. 2013;38:2-11 CrossRef Medline

4. Casselman JW, Kuhweide R, Deimling M, et al. Constructive interference in steady state-3DFT MR imaging of the inner ear and cerebellopontine angle. AJNR Am J Neuroradiol 1993;14:47-57 Medline

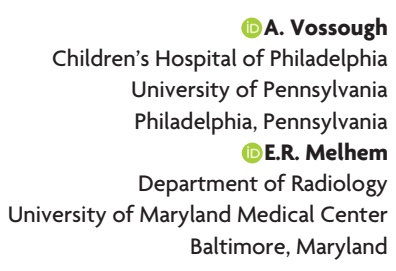

(1) A. Vossough University of Pennsylvania Philadelphia, Pennsylvania Department of Radiology Baltimore, Maryland 